\title{
GLUING FORMULAE OF SPECTRAL INVARIANTS AND CAUCHY DATA SPACES
}

\author{
JINSUNG PARK \\ School of Mathematics, Korea Institute for Advanced Study \\ 207-43, Cheongnyangni 2-dong, Dongdaemun-gu \\ Seoul 130-722, Korea \\ E-mail: jinsung@kias.re.kr
}

\begin{abstract}
We review the gluing formulae of the spectral invariants - the $\zeta$-regularized determinant of a Laplace type operator and the eta invariant of a Dirac type operator. In particular, we explain the crucial role of the Cauchy data spaces in these gluing formulae.

2000 MSC. Primary 58J28, 58J52.
\end{abstract}

\section{Introduction}

In this article, we survey the gluing formulae of the spectral invariants - the $\zeta$-regularized determinant of a Laplace type operator and the eta invariant of a Dirac type operator. After these spectral invariants had been originally introduced by Ray-Singer [30] and Atiyah-Patodi-Singer [1] respectively, these invariants have been studied by many people in many different parts of mathematics and physics. Here we discuss the gluing formulae of these spectral invariants. These formulae have been proved independently by several authors using different techniques. For nice introductions to this subject, we refer to Bleecker-Booß-Bavnbek [3] and Mazzeo-Piazza [21] where the reader can find many technical details and ideas of proofs. Therefore, instead of repeating the details of these introductions, we explain one principle which holds for all the known gluing formulae of the spectral invariants. This principle also enabled us to get a new proof of the gluing formulae of the eta invariant of a Dirac type operator and simultaneously to prove the gluing formula of the $\zeta$-regularized determinant of a Dirac Laplacian [17], [18]. We hope that this article would be helpful in the understanding of gluing formulae of the spectral invariants and other related gluing problems in similar situations.

Now let us review briefly the history of this subject: gluing problems 
of the spectral invariants. This will help the reader to understand the motivation of this article.

First, it is appropriate to begin with mentioning the pioneering work of Wojciechowski. In his paper with Douglas [10], they found a striking formula, which states that the eta invariant of a Dirac type operator over a manifold with boundary converges to a local expression as the cylindrical length near the boundary is getting longer and longer (the adiabatic limit). Although they did not formulate the gluing formula of the eta invariant in their paper [10] explicitly, this result suggested the existence of the gluing formula of the eta invariant.

The work of Douglas-Wojciechowski [10] stimulated many mathematicians working around the eta invariant, so after their paper appeared, during the last 15 years the gluing formula of the eta invariant has been proved independently and using different techniques by Hassell-MazzeoMelrose [12], Wojciechowski [34], Bunke [6], Müller [23], Brüning-Lesch [5], Kirk-Lesch [14], Park-Wojciechowski [27] and many others. Although their proofs are different from each other, they altogether used the generalized Atiyah-Patodi-Singer spectral projection to impose the boundary conditions for the Dirac operator over manifold with boundary. Among the aforementioned works, the formula of Kirk-Lesch is the most complete in the sense that their formula has no integer ambiguity (Bunke's formula also holds without the integer ambiguity) and they show the origin of the integer contribution in their proof. In fact, they needed to use the Calderón projector for the boundary condition to formulate their formula. Hence, this seems to suggest that the Calderón projector might be the natural projection in the gluing formula instead of the generalized Atiyah-Patodi-Singer spectral projection.

We can also see such a suggestion from the adiabatic decomposition formula of the $\zeta$-regularized determinant of the Dirac Laplacian proved by Park-Wojciechowski [25], [26], [27]. In their formula, the adiabatic limit of the ratio of the $\zeta$-regularized determinants of the Dirac Laplacians over the original manifold and decomposed submanifolds is mainly described in terms of the scattering matrices of the corresponding Dirac operators over manifolds with cylindrical ends, which are obtained by attaching half infinite cylinders to the decomposed manifolds with boundaries. Here, we can regard the scattering matrix for a noncompact manifold with cylindrical end as corresponding to the Calderón projector for a manifold with boundary.

Following this suggestion - the use of the Calderón projector, Loya and 
Park [17] could find a new proof of the gluing formula of the eta invariant of a Dirac type operator, which also provides us, simultaneously, with the gluing formula of the $\zeta$-regularized determinant of a Dirac Laplacian. Actually, these formulas are not two different formulas, but just two aspects - phase and modulus - of one unified formula. To state their formulae, they needed to introduce an operator $U$ which is defined by the Cauchy data spaces of the restricted Dirac operators over the decomposed manifolds. The proof in [17] can be easily employed for more general situations, for instance, for noncompact manifolds [18]. (Technically, the proof in [17] does not use the fact the variation of the eta invariant is locally computable, which holds only for compact manifolds.)

We can also see such a suggestion in the gluing formula of the $\zeta$ regularized determinant proved in the end of 80's by Burghelea-FriedlanderKappeler (BFK) [7]. In (a special case of) their formula, the ratio of the $\zeta$-regularized determinants of Laplace type operators over the original manifold and decomposed submanifolds is mainly described by the $\zeta$-regularized determinant of a certain operator $\mathcal{R}$ over the cutting hypersurface, and this operator $\mathcal{R}$ has an expression in terms of the Cauchy data spaces of the restricted Laplace operators over decomposed manifolds. As we will explain, the operators $U$ and $\mathcal{R}$ can be understood under the following principle: The gluing formulae of the spectral invariants are mainly described by the difference of the Cauchy data spaces.

Now let us explain the structure of this article. In Section 2, we first review the gluing formula of BFK [7] and one of its generalization [16]. In Section 3, we explain how the operator $\mathcal{R}$ can be understood in terms of the Cauchy data spaces. In Section 4, we review the gluing formulae of the spectral invariants of a Dirac type operator proved by Loya-Park [17]. Finally, in Section 5 , we explain the operator $U$, which plays the crucial role in the gluing formulae of the spectral invariants of a Dirac type operator, and its underlying meaning compared with $\mathcal{R}$.

The author gives his sincere thanks to Paul Loya for his helpful comments and the referee for corrections and suggestions, all of which considerably improved this article.

\section{Gluing formula of the $\zeta$-regularized determinant of a Laplace type operator}

In this section, we review the gluing formula of the $\zeta$-regularized determinant of a Laplace type operator. This type of formula was first proved 
by Burghelea, Friedlander and Kappeler (BFK) in [7] where they call this a Mayer-Vietoris type formula. Although their formula holds for a more general situation, that is, more general differential operators and general local elliptic boundary conditions, here we just restrict our discussion to a Laplace type operator and the Dirichlet boundary condition.

Now let us explain the BFK formula in more detail. Let $M$ is a compact manifold and $Y$ is a hypersurface in $M$, which decomposes $M$ into two submanifolds $M_{-}$and $M_{+}$(here we assume that $M_{-}$is the left side manifold and $M_{+}$is the right side manifold). Hence we have

$$
M=M_{-} \cup M_{+}, \quad Y=M_{-} \cap M_{+} .
$$

Let us consider a Laplace type operator over $M$,

$$
\Delta_{M}: H^{2}(M, E) \longrightarrow L^{2}(M, E),
$$

where $E$ is a Hermitian vector bundle over $M$. For the restrictions of $\Delta_{M}$ to $M_{-}$and $M_{+}$, we impose the Dirichlet boundary conditions so that we obtain

$$
\begin{aligned}
\Delta_{M_{ \pm}}:=\left.\Delta_{M}\right|_{M_{ \pm}}: \operatorname{dom}\left(\Delta_{M_{ \pm}}\right):=\left\{\phi \in H^{2}\left(M_{ \pm}, E\right) \mid\right. & \left.\gamma_{0}(\phi)=0\right\} \\
& \longrightarrow L^{2}\left(M_{ \pm}, E\right)
\end{aligned}
$$

where $\gamma_{0}: H^{2}\left(M_{ \pm}, E\right) \rightarrow H^{\frac{3}{2}}\left(Y, E_{0}\right)\left(E_{0}:=\left.E\right|_{Y}\right)$ denotes the restriction map to $Y$. Now let us recall that the $\zeta$-function of $\Delta_{M}$ is defined by

$$
\zeta\left(s, \Delta_{M}\right):=\frac{1}{\Gamma(s)} \int_{0}^{\infty} t^{s-1}\left[\operatorname{Tr}\left(e^{-t \Delta_{M}}\right)-\operatorname{dim} \operatorname{ker} \Delta_{M}\right] d t
$$

for $\Re s \gg 0$ and this has the meromorphic extension over $\mathbb{C}$ with $s=0$ as a regular value. Then the $\zeta$-regularized determinant of $\Delta_{M}$ is defined by

$$
\operatorname{det}_{\zeta} \Delta_{M}:=\exp \left(-\zeta^{\prime}\left(0, \Delta_{M}\right)\right)
$$

The $\zeta$-regularized determinant of $\Delta_{M_{ \pm}}$, $\operatorname{det}_{\zeta} \Delta_{M_{ \pm}}$is defined in a similar way as $\operatorname{det}_{\zeta} \Delta_{M}$. Now a natural question in this circumstance is the relation of $\operatorname{det}_{\zeta} \Delta_{M}$ with $\operatorname{det}_{\zeta} \Delta_{M_{-}}, \operatorname{det}_{\zeta} \Delta_{M_{+}}$, and the BFK formula gives us the answer to this question.

To explain their formula, we need to introduce an operator $\mathcal{R}$ acting on $C^{\infty}\left(Y, E_{0}\right)$, which is defined as follows: First, given $f \in C^{\infty}\left(Y, E_{0}\right)$, let us denote by $\phi_{ \pm}$the (assumed to be unique) solutions of the Dirichlet problems for the restrictions of $\Delta_{M}$ to $M_{ \pm}$:

$$
\Delta_{M} \phi_{i}=0 \quad \text { over } \quad M_{ \pm} \backslash Y,\left.\quad \phi_{ \pm}\right|_{Y}=f .
$$


Then, the operator $\mathcal{R}$ is defined by

$$
\mathcal{R} f:=\left.\left(\partial_{u} \phi_{-}\right)\right|_{Y}-\left.\left(\partial_{u} \phi_{+}\right)\right|_{Y}
$$

where $u$ is the normal variable to $Y$ such that $\pm \partial_{u}$ is the inward directional derivative for $M_{ \pm}$.

Remark 2.1. For $\mathcal{R}$ to be well defined, we need the condition that the Dirichlet problem of $\left.\Delta_{M}\right|_{M_{ \pm}}$has a unique solution. This is the case for the Laplace-Beltrami operator acting on the space of $k$-forms (see Remark 3.1). We always assume that the Laplace type operator $\Delta_{M}$ satisfies this condition in this article.

It is known that $\mathcal{R}$ is a nonnegative pseudodifferential operator over $Y$ of order 1 , hence its $\zeta$-regularized determinant is well defined. Now we are ready to state the $\mathrm{BFK}$ formula.

Theorem 2.1. [7] When $\operatorname{ker} \Delta_{M}=\{0\}$, we have

$$
\frac{\operatorname{det}_{\zeta} \Delta_{M}}{\operatorname{det}_{\zeta} \Delta_{M_{+}} \cdot \operatorname{det}_{\zeta} \Delta_{M_{-}}}=C(Y) \cdot \operatorname{det}_{\zeta} \mathcal{R}
$$

where $C(Y)$ is a constant depending only on the symbols of $\Delta_{M}, \Delta_{M_{ \pm}}, \mathcal{R}$ over $Y$.

The BFK formula in (3) describes the ratio of $\operatorname{det}_{\zeta} \Delta_{M}$ and $\operatorname{det}_{\zeta} \Delta_{M_{+}}$. $\operatorname{det}_{\zeta} \Delta_{M_{-}}$in terms of $\operatorname{det}_{\zeta} \mathcal{R}$ modulo the constant $C(Y)$, which can be considered as data near $Y$. Note that although the operator $\mathcal{R}$ is defined over $Y, \mathcal{R}$ contains global information over $M$ via the null solutions of the restrictions of $\Delta_{M}$ to $M_{ \pm}$.

Remark 2.2. By definition of $\mathcal{R}$, $\operatorname{ker} \Delta_{M}=\{0\}$ implies that $\operatorname{ker} \mathcal{R}=\{0\}$. Hence, under this condition, all the operators occurring in (3) have trivial kernels. Without this condition, we have an additional term on the right side of $(3)$.

Remark 2.3. When we assume that $\Delta_{M}$ has the following product form over a collar neighborhood $\mathcal{U} \cong Y \times[-1,1]_{u}$ of $Y$,

$$
\left.\Delta_{M}\right|_{\mathcal{U}}=-\frac{d^{2}}{d u^{2}}+\Delta_{Y}
$$

where $u$ denotes the variable of the normal direction to $Y$ and $\Delta_{Y}$ is a Laplace type operator over $Y$, we can obtain the exact value of $C(Y)$ as in $[9],[15],[28]$,

$$
C(Y)=2^{-\zeta\left(0, \Delta_{Y}\right)-h_{Y}}
$$


Here $\zeta\left(s, \Delta_{Y}\right)$ is the $\zeta$-function of $\Delta_{Y}$ and $h_{Y}:=\operatorname{dim} \operatorname{ker} \Delta_{Y}$.

For a noncompact manifold $M$, the operator $e^{-t \Delta_{M}}$ is not of trace class. Hence the $\zeta$-regularized determinant cannot be defined as in compact case. But, in this case, one can use the $b$-trace of Melrose [22] or the relative trace of Müller [24] instead of using the ordinary trace of $e^{-t \Delta_{M}}$. For the $\zeta$-regularized determinant defined by the $b$-trace or relative trace over a noncompact manifold, its gluing formulae have been proved by HassellZelditch [13] for the decomposition of $M=\mathbb{R}^{2}$ into a compact smooth domain and its complement, and by Carron [9] for the general noncompact case. Here we just explain one generalization of the BFK formula in (3) for a noncompact manifold $X$ with cylindrical end. The manifold $X$ with cylindrical end has the following decomposition,

$$
X=N \cup_{Y} Z
$$

where $N$ is a manifold with boundary $Y$ and $Z \cong Y \times[0, \infty)_{u}$. We may assume there is a collar neighborhood $W \cong Y \times[-1,0]_{u}$ of $Y$ within $N$. We consider a Laplace type operator $\Delta_{X}$ acting on $C^{\infty}(X, E)$ where $E$ is a Hermitian vector bundle over $X$. We also assume product structures of the Riemannian metric of $X$ and the Hermitian metric of $E$ over $W \cup_{Y} Z \cong$ $Y \times[-1, \infty)_{u}$. Finally we assume the following expression of $\Delta_{X}$ over $W \cup_{Y} Z$,

$$
\left.\Delta_{X}\right|_{W \cup_{Y} Z}=-\frac{d^{2}}{d u^{2}}+\Delta_{Y}
$$

where $\Delta_{Y}$ is a Laplace type operator over $Y$. As before, we impose the Dirichlet boundary conditions for the restrictions of $\Delta_{X}$ to $N$ and $Z$ and denote by $\Delta_{N}, \Delta_{Z}$ the resulting operators. Then the relative $\zeta$-function for $\left(\Delta_{X}, \Delta_{Z}\right)$ is defined by

$$
\zeta\left(s, \Delta_{X}, \Delta_{Z}\right):=\frac{1}{\Gamma(s)}\left(\int_{0}^{1}+\int_{1}^{\infty}\right) t^{s-1} \operatorname{Tr}\left(e^{-t \Delta_{X}}-e^{-t \Delta_{Z}}\right) d t .
$$

Here the integral $\int_{0}^{1} \cdot d t$ has a meromorphic extension from $\Re s \gg 0$ to $\mathbb{C}$ and the integral $\int_{1}^{\infty} \cdot d t$ has a meromorphic extension from $\Re s \ll 0$ to $\mathbb{C}$. The resulting meromorphic extension of $\zeta\left(s, \Delta_{X}, \Delta_{Z}\right)$ is regular at $s=0$. (In the above definition of $\zeta\left(s, \Delta_{X}, \Delta_{Z}\right)$, the relative trace $\operatorname{Tr}\left(e^{-t \Delta_{X}}-e^{-t \Delta_{Z}}\right)$ contains the zero eigenvalues of $\Delta_{X}$, but these are cancelled out after taking the sum of $\int_{0}^{1} \cdot d t$ and $\int_{1}^{\infty} \cdot d t$.) Then the relative $\zeta$-regularized determinant is defined by

$$
\operatorname{det}_{\zeta}\left(\Delta_{X}, \Delta_{Z}\right):=\exp \left(-\zeta^{\prime}\left(0, \Delta_{X}, \Delta_{Z}\right)\right)
$$


To explain the generalization of the BFK formula to manifolds with cylindrical end, we need to introduce some more notations. Let $\left\{u_{j}\right\}$ be an orthonormal basis for the kernel of $\Delta_{X}$ on $L^{2}(X, E)$ and let $\left\{U_{j}\right\}$ be a basis of the 'extended $L^{2}$-solutions' (bounded solutions of $\Delta_{X} U_{j}=0$ ) such that at $\infty$ on the cylinder, $\left\{U_{j}(\infty)\right\}$ are orthonormal in $L^{2}\left(Y, E_{0}\right)$ where $E_{0}:=\left.E\right|_{Y}$. Let $v_{j}=\left.u_{j}\right|_{Y}$ and $V_{j}=\left.U_{j}\right|_{Y}$ be the restrictions of $u_{j}$ and $U_{j}$, respectively, to the hypersurface $\{0\} \times Y$. It can be shown that the sections $\left\{v_{j}, V_{j}\right\}$ are linearly independent in $L^{2}\left(Y, E_{0}\right)$, therefore both operators

$$
L=\sum_{j} v_{j} \otimes v_{j}^{*}, \quad \widetilde{L}=\sum_{j} V_{j} \otimes V_{j}^{*}
$$

are nonnegative linear operators on the finite-dimensional vector space $V=$ $\operatorname{span}\left\{v_{j}, V_{j}\right\} \subset L^{2}\left(Y, E_{0}\right)$. Since the set $\left\{v_{j}, V_{j}\right\}$ is a linearly independent set spanning $V$, the operator

$$
L+\widetilde{L}: V \longrightarrow V
$$

is positive. In particular, $\operatorname{det}(L+\widetilde{L})$ is nonzero. Now we can state the main result of $[16]$.

Theorem 2.2. [16] The following equality holds,

$$
\frac{\operatorname{det}_{\zeta}\left(\Delta_{X}, \Delta_{Z}\right)}{\operatorname{det}_{\zeta} \Delta_{N}}=2^{-\zeta\left(0, \Delta_{Y}\right)-h_{Y}} \frac{\operatorname{det}_{\zeta} \mathcal{R}}{\operatorname{det}(L+\widetilde{L})}
$$

where $\zeta\left(s, \Delta_{Y}\right)$ is the $\zeta$-function of $\Delta_{Y}$ and $h_{Y}=\operatorname{dim} \operatorname{ker} \Delta_{Y} \cdot{ }^{\mathrm{a}}$

Remark 2.4. Originally the formula (6) was given in terms of the $b$-trace in [16]. We refer to [16] for this and an elementary introduction to the $b$-trace. Since we do not assume any condition on the kernel of $\Delta_{X}$, we have the additional term $\operatorname{det}(L+\widetilde{L}$ ) on the right side of (6) (see Remark 2.2 ). By the product structure in (5), we could obtain the explicit form of the constant $C(Y)=2^{-\zeta\left(0, \Delta_{Y}\right)-h_{Y}}$ as in (4).

\section{The operator $\mathcal{R}$ and the Cauchy data spaces of Laplace type operator}

The discussion in this section holds for a more general situation, but we just restrict our concern to the closed manifold $M$. The main purpose of

a After [16] was completed, we learned that this result was also proved in "Regularized determinants of Laplace type operators, analytic surgery and relative determinants" by J. Müller and W. Müller. 
this section is to investigate the operator $\mathcal{R}$ in terms of the Cauchy data spaces of $\left.\Delta_{M}\right|_{M_{ \pm}}$.

Now we recall the operator $\Delta_{M}$ over $M$ which is decomposed into $M_{-}, M_{+}$by the hypersurface $Y$. The trace map $\gamma$ is defined by

$$
\gamma(\phi)=\left(\left.\phi\right|_{Y},\left.\left(\partial_{u} \phi\right)\right|_{Y}\right): H^{2}(M, E) \longrightarrow H^{\frac{3}{2}}\left(Y, E_{0}\right) \oplus H^{\frac{1}{2}}\left(Y, E_{0}\right)
$$

where $E_{0}:=\left.E\right|_{Y}$. Here $u$ denotes the normal variable for the collar neighborhood $\mathcal{U} \cong Y \times[-1,1]_{u}$ of $Y$ and $\pm \partial_{u}$ is the inward directional normal derivative to $M_{ \pm}$. Then the restriction $\Delta_{ \pm}:=\left.\Delta_{M}\right|_{M_{ \pm}}$determines the Cauchy data space $\mathcal{H}\left(\Delta_{ \pm}\right)$defined by

$$
\begin{aligned}
& \mathcal{H}\left(\Delta_{ \pm}\right):=\left\{(f, g) \in C^{\infty}\left(Y, E_{0}\right) \oplus C^{\infty}\left(Y, E_{0}\right) \mid \exists \phi \in C^{\infty}\left(M_{ \pm}, E\right)\right. \\
&\text { such that } \left.\Delta_{M} \phi=0 \text { on } M_{ \pm} \backslash Y \text { and } \gamma(\phi)=(f, g)\right\} .
\end{aligned}
$$

Hence, $\mathcal{H}\left(\Delta_{ \pm}\right)$consists of the pair of the Dirichlet and Neumann data of $\Delta_{ \pm}$over $M_{ \pm}$.

The Dirichlet-to-Neumann operator $\mathcal{N}_{ \pm}$over $C^{\infty}\left(Y, E_{0}\right)$ is defined to be the map sending $f \in C^{\infty}\left(Y, E_{0}\right)$ to the corresponding Neumann data $g \in C^{\infty}\left(Y, E_{0}\right)$ such that $(f, g) \in \mathcal{H}\left(\Delta_{ \pm}\right)$. Note that the well-definedness of $\mathcal{N}_{ \pm}\left(\operatorname{ker} \mathcal{N}_{ \pm}=\{0\}\right)$ is equivalent to the condition that the operator $\Delta_{ \pm}$ with the Dirichlet (Neumann) boundary condition has no null solution.

Remark 3.1. Let us consider the case of the Laplace-Beltrami operator $\left(d+d^{*}\right)^{2}$ acting on the space of $k$-forms over a manifold with boundary. The operator $\left(d+d^{*}\right)^{2}$ with the Dirichlet boundary condition has no null solution. Indeed, a null solution of $\left(d+d^{*}\right)^{2}$ would also be a null solution of $d+d^{*}$ by the Green formula. But, this is impossible by the unique continuation theorem for $d+d^{*}[2]$. Hence, the Dirichlet-to-Neumann operator for the Laplace-Beltrami operator is well defined.

Under the condition assumed in Remark 2.1, the operator $\mathcal{N}_{ \pm}$defines its graph which is exactly $\mathcal{H}\left(\Delta_{ \pm}\right)$. We refer to [29], [20] for some more detailed explanations about the Dirichlet-to-Neumann operator and its application to the relative formula of the Dirichlet/Neumann Laplacians.

Now, recalling the definition of $\mathcal{R}$ and $\mathcal{N}_{ \pm}$, we can easily see that

$$
\mathcal{R}=\mathcal{N}_{-}-\mathcal{N}_{+}: C^{\infty}\left(Y, E_{0}\right) \longrightarrow C^{\infty}\left(Y, E_{0}\right)
$$

Hence, we can see that the operator $\mathcal{R}$ describes the difference of two Cauchy data spaces $\mathcal{H}\left(\Delta_{ \pm}\right)$in the sense that

$$
(f, \mathcal{R} f)=\left(f, \mathcal{N}_{-} f\right)-\left(f, \mathcal{N}_{+} f\right) \quad \text { for } \quad\left(f, \mathcal{N}_{ \pm} f\right) \in \mathcal{H}\left(\Delta_{ \pm}\right) .
$$




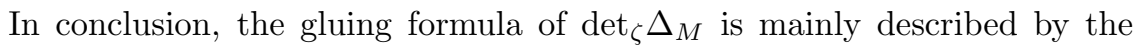
difference of the Cauchy data spaces $\mathcal{H}\left(\Delta_{ \pm}\right)$.

\section{Gluing formula of the spectral invariants of a Dirac type operator}

In this section, we discuss the gluing formulae [17] of the spectral invariants of Dirac type operators, that is, the eta invariant of a Dirac type operator and the $\zeta$-regularized determinant of a Dirac Laplacian.

Let $\mathcal{D}$ be a Dirac type operator acting on $C^{\infty}(M, S)$ where $M$ is a closed compact Riemannian manifold of arbitrary dimension and $S$ is a Clifford bundle over $M$. Let $Y$ be an embedded hypersurface in $M$ which decomposes $M$ into two submanifolds $M_{-}$and $M_{+}$. Hence we have

$$
M=M_{-} \cup M_{+}, \quad Y=M_{-} \cap M_{+} .
$$

We assume all geometric structures are of product type over a tubular neighborhood $\mathcal{U} \cong[-1,1]_{u} \times Y$ of $Y$ where the Dirac operator takes the product form over $\mathcal{U}$,

$$
\left.\mathcal{D}\right|_{\mathcal{U}}=G\left(\partial_{u}+D_{Y}\right)
$$

where $G$ is a unitary operator on $S_{0}:=\left.S\right|_{Y}$ and $D_{Y}$ is a Dirac type operator over $Y$ satisfying $G^{2}=-\mathrm{Id}$ and $D_{Y} G=-G D_{Y}$. Recall that the eta function of $\mathcal{D}$ and the zeta function of $\mathcal{D}^{2}$ are defined through the heat operator $e^{-t \mathcal{D}^{2}}$ by

$$
\begin{gathered}
\eta(s, \mathcal{D}):=\frac{1}{\Gamma\left(\frac{s+1}{2}\right)} \int_{0}^{\infty} t^{\frac{s-1}{2}} \operatorname{Tr}\left(\mathcal{D} e^{-t \mathcal{D}^{2}}\right) d t \\
\zeta\left(s, \mathcal{D}^{2}\right):=\frac{1}{\Gamma(s)} \int_{0}^{\infty} t^{s-1}\left[\operatorname{Tr}\left(e^{-t \mathcal{D}^{2}}\right)-\operatorname{dim} \operatorname{ker} \mathcal{D}\right] d t,
\end{gathered}
$$

which are defined a priori for $\Re s \gg 0$ and extend to be meromorphic functions on $\mathbb{C}$ that are regular at $s=0$. The eta invariant and the reduced eta invariant of $\mathcal{D}$ are defined by

$$
\eta(\mathcal{D}):=\eta(0, \mathcal{D}), \quad \tilde{\eta}(\mathcal{D}):=\frac{1}{2}(\eta(0, \mathcal{D})+\operatorname{dim} \operatorname{ker} \mathcal{D}),
$$

and the $\zeta$-determinant of $\mathcal{D}^{2}, \operatorname{det}_{\zeta} \mathcal{D}^{2}$ is defined as in $(2) \operatorname{using} \zeta\left(s, \mathcal{D}^{2}\right)$.

By restriction, $\mathcal{D}$ induces Dirac type operators $\mathcal{D}_{ \pm}$over $M_{ \pm}$. For these operators, we choose orthogonal projections $\mathcal{P}_{ \pm}$over $L^{2}\left(Y, S_{0}\right)$ that provide 
us with well-posed boundary conditions for $\mathcal{D}_{ \pm}$in the sense of Seeley [33]. Then the following operators

$$
\mathcal{D}_{\mathcal{P}_{ \pm}}: \operatorname{dom}\left(\mathcal{D}_{\mathcal{P}_{ \pm}}\right) \rightarrow L^{2}\left(M_{ \pm}, S\right)
$$

where

$$
\operatorname{dom}\left(\mathcal{D}_{\mathcal{P}_{ \pm}}\right):=\left\{\phi \in H^{1}\left(M_{ \pm}, S\right) \mid \mathcal{P}_{ \pm}\left(\left.\phi\right|_{Y}\right)=0\right\}
$$

share many of the analytic properties of $\mathcal{D}$; in particular, they are Fredholm and have discrete spectra, but are not necessarily self-adjoint. Amongst such projectors are the (orthogonalized) Calderón projectors $\mathcal{C}_{ \pm}$[8], [32] which are projectors defined intrinsically as the unique orthogonal projectors onto the closures in $L^{2}\left(Y, S_{0}\right)$ of the infinite-dimensional Cauchy data spaces of $\mathcal{D}_{ \pm}$:

$$
\begin{aligned}
\mathcal{H}\left(\mathcal{D}_{ \pm}\right):=\left\{f \in C^{\infty}\left(Y, S_{0}\right) \mid \exists \phi \in C^{\infty}\left(M_{ \pm}, S\right)\right. \text { such that } \\
\left.\mathcal{D} \phi=0 \text { on } M_{ \pm} \backslash Y \text { and }\left.\phi\right|_{Y}=f\right\} .
\end{aligned}
$$

To state our main theorem, we recall that the Calderón projectors $\mathcal{C}_{ \pm}$ have the matrix forms

$$
\mathcal{C}_{ \pm}=\frac{1}{2}\left(\begin{array}{cc}
\mathrm{Id} & \kappa_{ \pm}^{-1} \\
\kappa_{ \pm} & \mathrm{Id}
\end{array}\right)
$$

with respect to the decomposition $L^{2}\left(Y, S_{0}\right)=L^{2}\left(Y, S^{+}\right) \oplus L^{2}\left(Y, S^{-}\right)$, where $S^{ \pm} \subset S_{0}$ are the $( \pm i)$-eigenspaces of $G$. The maps

$$
\kappa_{ \pm}: L^{2}\left(Y, S^{+}\right) \rightarrow L^{2}\left(Y, S^{-}\right)
$$

are isometries, so that $U:=-\kappa_{-} \kappa_{+}^{-1}$ is a unitary operator over $L^{2}\left(Y, S^{-}\right)$, which is moreover of Fredholm determinant class. The last property follows easily from the fact that the differences of $\mathcal{C}_{ \pm}$and the Atiyah-Patodi-Singer spectral projections are smoothing operators; we refer to [31] for the details. We denote by $\widehat{U}$ the restriction of $U$ to the orthogonal complement of its (-1)-eigenspace. We also put

$$
L:=\sum_{j=1}^{h_{M}} v_{j} \otimes v_{j}^{*}
$$

where $h_{M}=\operatorname{dim} \operatorname{ker} \mathcal{D}$ and $v_{j}=\left.u_{j}\right|_{Y}$ with the orthonormal basis $\left\{u_{j}\right\}$ of $\operatorname{ker} \mathcal{D}$. Then $L$ is a positive operator on the finite-dimensional vector space $\left.(\operatorname{ker} \mathcal{D})\right|_{Y}$. We are now ready to introduce the main result of [17]. 
Theorem 4.1. [17] The following gluing formulae hold:

$$
\begin{gathered}
\frac{\operatorname{det}_{\zeta} \mathcal{D}^{2}}{\operatorname{det}_{\zeta} \mathcal{D}_{\mathcal{C}_{-}}^{2} \cdot \operatorname{det}_{\zeta} \mathcal{D}_{\mathcal{C}_{+}}^{2}}=2^{-\zeta\left(0, D_{Y}^{2}\right)-h_{Y}}(\operatorname{det} L)^{-2} \operatorname{det}_{F}\left(\frac{2 \operatorname{Id}+\widehat{U}+\widehat{U}^{-1}}{4}\right), \\
\tilde{\eta}(\mathcal{D})-\tilde{\eta}\left(\mathcal{D}_{\mathcal{C}_{-}}\right)-\tilde{\eta}\left(\mathcal{D}_{\mathcal{C}_{+}}\right)=\frac{1}{2 \pi i} \log \operatorname{det}_{F} U \bmod \mathbb{Z},
\end{gathered}
$$

where $\zeta\left(s, D_{Y}^{2}\right)$ is the $\zeta$-function of $D_{Y}^{2}, h_{Y}=\operatorname{dim} \operatorname{ker} D_{Y}$, $\operatorname{det}_{F}$ denotes the Fredholm determinant, and Log the principal value logarithm.

We can replace the Calderón projector $\mathcal{C}_{ \pm}$in the gluing formulae in Theorem 4.1 by other orthogonal projection in the smooth, self-adjoint Grassmannian $G r_{\infty}^{*}\left(\mathcal{D}_{ \pm}\right)$, which consists of orthogonal projections $\mathcal{P}_{ \pm}$such that $\mathcal{P}_{ \pm}-\mathcal{C}_{ \pm}$are smoothing operators and $G \mathcal{P}_{ \pm}=\left(\mathrm{Id}-\mathcal{P}_{ \pm}\right) G$. Let $\mathcal{P}_{1} \in$ $G r_{\infty}^{*}\left(\mathcal{D}_{-}\right)$and $\mathcal{P}_{2} \in G r_{\infty}^{*}\left(\mathcal{D}_{+}\right)$. Then the eta invariant of $\mathcal{D}_{\mathcal{P}_{i}}$ and the $\zeta$ regularized determinant of $\mathcal{D}_{\mathcal{P}_{i}}^{2}$ are well defined by the results of Grubb [11] and Wojciechowski [35]. The orthogonal projections $\mathcal{P}_{1}$ and $\mathcal{P}_{2}$ determine maps $\kappa_{1}$ and $\kappa_{2}$ as in (10), and we define

$$
U_{1}:=\kappa_{-} \kappa_{1}^{-1}, \quad U_{2}:=\kappa_{2} \kappa_{+}^{-1}, \quad U_{12}:=-\kappa_{1} \kappa_{2}^{-1} \quad \text { over } L^{2}\left(Y, S^{-}\right) .
$$

As before, let us denote by $\widehat{U}_{i}$ the restriction of $U_{i}$ to the orthogonal complement of its $(-1)$-eigenspace. We define the operator $L_{1}$ over the finitedimensional vector space $\operatorname{ran}\left(\mathcal{C}_{-}\right) \cap \operatorname{ran}\left(\mathrm{Id}-\mathcal{P}_{1}\right)$ by

$$
L_{1}=-P_{1} G \mathcal{R}_{-}^{-1} G P_{1}
$$

where $\mathcal{R}_{-}$is the BFK operator for the double of $\left(\mathcal{D}_{-}\right)^{2}$ (see chapter 9 of [4] for the double construction), and $P_{1}$ is the orthogonal projection onto $\operatorname{ran}\left(\mathcal{C}_{-}\right) \cap \operatorname{ran}\left(\operatorname{Id}-\mathcal{P}_{1}\right)$. Then $L_{1}$ is a positive operator [19]. We define $L_{2}$ in a similar way. We can now state the general gluing formulae for the spectral invariants of Dirac type operators.

Theorem 4.2. [17] The following general gluing formulae hold:

$$
\begin{aligned}
& \frac{\operatorname{det}_{\zeta} \mathcal{D}^{2}}{\operatorname{det}_{\zeta} \mathcal{D}_{\mathcal{P}_{1}}^{2} \cdot \operatorname{det}_{\zeta} \mathcal{D}_{\mathcal{P}_{2}}^{2}}=2^{-\zeta\left(0, D_{Y}^{2}\right)-h_{Y}}(\operatorname{det} L)^{-2} \operatorname{det}_{F}\left(\frac{2 \operatorname{Id}+\widehat{U}+\widehat{U}^{-1}}{4}\right) \\
& \prod_{i=1}^{2}\left(\operatorname{det} L_{i}\right)^{-2} \operatorname{det}_{F}\left(\frac{2 \mathrm{Id}+\widehat{U}_{i}+\widehat{U}_{i}^{-1}}{4}\right)^{-1} \\
& \tilde{\eta}(\mathcal{D})-\tilde{\eta}\left(\mathcal{D}_{\mathcal{P}_{1}}\right)-\tilde{\eta}\left(\mathcal{D}_{\mathcal{P}_{2}}\right)=\frac{1}{2 \pi i} \log \operatorname{det}_{F} U_{12} \bmod \mathbb{Z}
\end{aligned}
$$


Remark 4.1. As in Theorem 2.2, we can generalize Theorem 4.2 to noncompact manifolds with cylindrical end. We refer to [18] for this result and its proof.

The gluing formula of the eta invariant in Theorem 4.2 (when $\mathcal{P}_{i}$ are the generalized Atiyah-Patodi-Singer spectral projections) has the same form as (or its reduced form modulo $\mathbb{Z}$ of) the gluing formulae proved by Hassell-Mazzeo-Melrose [12], Wojciechowski [34], Bunke [6], Müller [23], Brüning-Lesch [5], Kirk-Lesch [14], Park-Wojciechowski [27]. In this formula, the data given by the Calderón projector or the Cauchy data spaces are cancelled so these data do not appear in the gluing formula. This is the main reason that the important role of the Cauchy data spaces in the gluing formula of the eta invariant has not been noticed before the work of Kirk-Lesch [14]. But, in the gluing formula of the $\zeta$-regularized determinant in Theorem 4.2, these terms appear via $\widehat{U}$ with the additional terms $\widehat{U}_{i}$ when we impose boundary conditions given by orthogonal projections other than the Calderón projectors $\mathcal{C}_{ \pm}$. Comparing the gluing formulae in Theorems 4.1 and 4.2, we can see that the Calderón projectors are the most natural projections for the gluing formulae of the spectral invariants since imposing the boundary conditions by these projections makes the formulae the simplest possible.

\section{The operator $U$ and the Cauchy data spaces of a Dirac type operator}

In this section, we investigate the operator $U$, which appears both gluing formulae of the $\zeta$-regularized determinant and the eta invariant in Theorem 4.1. The purpose of this section is to explain that the unitary operator $U$ also describes the difference of the Cauchy data spaces $\mathcal{H}\left(\mathcal{D}_{ \pm}\right)$as $\mathcal{R}$ did the difference of $\mathcal{H}\left(\Delta_{ \pm}\right)$.

As before, let us consider the operator $\mathcal{D}$ over the closed manifold $M$ which is decomposed into $M_{-}, M_{+}$by the hypersurface $Y$. (The following discussion holds for more general situations, but we just restrict our concern to the closed manifold $M$.) Now let us recall that $\mathcal{H}\left(\mathcal{D}_{ \pm}\right)$consists of the boundary values of the null-solutions of $\mathcal{D}_{ \pm}$over $M_{ \pm}$(see the definition (9)) and the (orthogonalized) Calderón projectors $\mathcal{C}_{ \pm}$are the unique orthogonal projectors onto the closures of $\mathcal{H}\left(\mathcal{D}_{ \pm}\right), \overline{\mathcal{H}\left(\mathcal{D}_{ \pm}\right)}$in $L^{2}\left(Y, S_{0}\right)$.

To explain the underlying meaning of $U$, let us explain how we could derive the definition of the operator $U$ by modelling on the operator $\mathcal{R}$.

First, let us recall the expression of $\mathcal{R}$ in (7) with $\mathcal{N}_{ \pm}$and that $\mathcal{N}_{ \pm}$ 
determines $\mathcal{H}\left(\Delta_{ \pm}\right)$as its graph. So, our task is to find the operator whose graph is $\mathcal{H}\left(\mathcal{D}_{ \pm}\right)$. Noting that the Calderón projector $\mathcal{C}_{ \pm}$has the matrix form in (10), we can see that $\kappa_{ \pm}$determines (the closure in $L^{2}\left(Y, S_{0}\right)$ of) $\mathcal{H}\left(\mathcal{D}_{ \pm}\right)$as its graph.

Now the question is how we define $U$ using $\kappa_{ \pm}$. We here observe that $\overline{\mathcal{H}\left(\mathcal{D}_{ \pm}\right)}$is a Lagrangian subspace in $L^{2}\left(Y, S_{0}\right)$ with respect to the symplectic form $\langle G$,$\rangle . Hence, there is the unitary operator over L^{2}\left(Y, S^{-}\right)$which transforms $\mathcal{H}\left(\mathcal{D}_{+}\right)$to $\mathcal{H}\left(\mathcal{D}_{-}\right)$, that is, describes the difference of them. From this reasoning, we can see that the operator $\kappa_{-} \kappa_{+}^{-1}$ does this since

$$
\left(x, \kappa_{-} x\right)=\left(x,\left(\kappa_{-} \kappa_{+}^{-1}\right) \kappa_{+} x\right) \quad \text { for } \quad\left(x, \kappa_{ \pm} x\right) \in \mathcal{H}\left(\mathcal{D}_{ \pm}\right) .
$$

But, we actually need to find the unitary operator which transforms the Cauchy data space of $\mathcal{D}_{+}^{*}$ to $\mathcal{H}\left(\mathcal{D}_{-}\right)$, where $\mathcal{D}_{+}^{*}$ is the reflection of the Dirac type operator $\mathcal{D}_{+}$to the manifold $M_{+}^{*}$, which is the left side manifold on the double of $M_{+}$. This is because $M_{+}\left(M_{-}\right)$is a right (left) side manifold and we have to compare the Cauchy data spaces over the left side manifolds to measure the true difference of the Cauchy data spaces. Recalling the double construction in chapter 9 of [4], we can see that the corresponding Calderón projector $\mathcal{C}_{+}^{*}$ on the left side manifold $M_{+}^{*}$ to $\mathcal{C}_{+}$on $M_{+}$is given by

$$
\mathrm{Id}-\mathcal{C}_{+}=\frac{1}{2}\left(\begin{array}{cc}
\mathrm{Id} & -\kappa_{+}^{-1} \\
-\kappa_{+} & \mathrm{Id}
\end{array}\right)
$$

Therefore, the operator $-\kappa_{+}$determines the Cauchy data space $\mathcal{H}\left(\mathcal{D}_{+}^{*}\right)$ as its graph. In conclusion, we can see that $U=-\kappa_{-} \kappa_{+}^{-1}$ is the correct operator measuring the true difference of the Cauchy data spaces.

The interesting point is that we can obtain the modulus and the phase data from $U$ via the following equality:

$$
\left(\frac{\mathrm{Id}+U}{2}\right)^{2}=U\left(\frac{2 \mathrm{Id}+U+U^{-1}}{4}\right),
$$

where the principal $\operatorname{logarithm}$ of the first factor $U, \log U$ describes the gluing formula of the eta invariant and the second modulus part describes the gluing formula of the $\zeta$-regularized determinant in Theorem 4.1. In fact, this is not a simple coincidence but follows from the deep relation between the eta invariant of the Dirac operator and the $\zeta$-regularized determinant of the Dirac Laplacian as the proof of Theorem 4.1 shows. We refer to [17] for the details of its proof. 


\section{References}

1. M.F. Atiyah, V.K. Patodi, and I.M. Singer, Spectral asymmetry and Riemannian geometry. I, Math. Proc. Cambridge Philos. Soc. 77 (1975), 43-69.

2. C. Bär, Zero sets of solutions to semilinear elliptic systems of first order, Invent. Math. 138 (1999), 183-202.

3. D. Bleecker and B. Booß-Bavnbek, Spectral invariants of operators of Dirac type on partitioned manifolds, Aspects of Boundary Problems in Analysis and Geometry, 1-130, Oper. Theory Adv. Appl., 151, Birkhauser, Basel, 2004.

4. B. Booß-Bavnbek and K. P. Wojciechowski, Elliptic Boundary Problems for Dirac Operators, Birkhäuser Boston Inc., Boston, MA, 1993.

5. J. Brüning and M. Lesch, On the $\eta$-invariant of certain nonlocal boundary value problems, Duke Math. J. 96 (1999), 425-468.

6. U. Bunke, On the gluing formula for the $\eta$-invariant, J. Differential Geom. 41 (1995), 397-448.

7. D. Burghelea, L. Friedlander, and T. Kappeler, Mayer-Vietoris type formula for determinants of differential operators, J. Funct. Anal. 107 (1992), 34-65.

8. A.-P. Calderón, Boundary value problems for elliptic equations, 1963 Outlines Joint Sympos. Partial Differential Equations (Novosibirsk, 1963) pp. 303-304 Acad. Sci. USSR Siberian Branch, Moscow

9. G. Carron, Déterminant relatif et la fonction Xi, Amer. J. Math. 124, no. 2 (2002), 307-352.

10. R. G. Douglas and K. P. Wojciechowski: Adiabatic limits of the $\eta$-invariants. The odd-dimensional Atiyah-Patodi-Singer problem, Comm. Math. Phys. 142 (1991), 139-168.

11. G. Grubb, Poles of zeta and eta functions for perturbations of the AtiyahPatodi-Singer problem, Comm. Math. Phys. 215 (2001), 583-589.

12. A. Hassell, R. R. Mazzeo, and R. B. Melrose, Analytic surgery and the accumulation of eigenvalues, Comm. Anal. Geom. 3 (1995), 115-222.

13. A. Hassell and S. Zelditch, Determinants of Laplacians in exterior domains, IMRN 18 (1999), 971-1004.

14. P. Kirk and M. Lesch, The eta invariant, Maslov index, and spectral flow for Dirac-type operators on manifolds with boundary, Forum Math. 16 (2004), $553-629$.

15. Y. Lee, Burghelea-Friedlander-Kappeler's gluing formula for the zetadeterminant and its applications to the adiabatic decompositions of the zetadeterminant and the analytic torsion, Trans. Amer. Math. Soc. 355, no. 10 (2003), 4093-4110.

16. P. Loya and J. Park, Decomposition of the zeta-determinant for the Laplacian on manifolds with cylindrical end, Illinois J. Math. 48, no. 4 (2004), 1279-1303.

17. P. Loya and J. Park, On the gluing problem for the spectral invariants of Dirac operators, Advances in Math., to appear.

18. P. Loya and J. Park, On the gluing problem for Dirac operators on manifolds with cylindrical ends, J. Geom. Anal. 15 (2005), 285-319.

19. P. Loya and J. Park, The comparison problem for the spectral invariants of Dirac type operators, Preprint, 2004. 
20. P. Loya and J. Park, $\zeta$-determinants of Laplacians with Neumann and Dirichlet boundary conditions, J. Phys. A, 38 (2005), 8967-8977.

21. R. Mazzeo and P. Piazza, Dirac operators, heat kernels and microlocal analysis. II. Analytic surgery, Rend. Mat. Appl. (7) 18, no. 2 (1998), 221-288.

22. R. B. Melrose, The Atiyah-Patodi-Singer Index Theorem, A.K. Peters, Wellesley, 1993.

23. W. Müller, On the $L^{2}$-index of Dirac operators on manifolds with corners of codimension two. I, J. Differential Geom. 44 (1996), 97-177.

24. W. Müller, Relative zeta functions, relative determinants and scattering theory, Comm. Math. Phys. 192 (1998), 309-347.

25. J. Park and K. P. Wojciechowski, Adiabatic decomposition of the $\zeta$ determinant of the Dirac Laplacian. I. The case of an invertible tangential operator. With an appendix by Yoonweon Lee, Comm. Partial Differential Equations 27 (2002), no. 7-8, 1407-1435.

26. J. Park and K. P. Wojciechowski, Scattering theory and adiabatic decomposition of the $\zeta$-determinant of the Dirac Laplacian, Math. Res. Lett. 9 (2002), no. $1,17-25$.

27. J. Park and K. P. Wojciechowski, Adiabatic decomposition of the $\zeta$-determinant and Scattering theory, Michigan Math. J., to appear, math.DG/0111046 .

28. J. Park and K. P. Wojciechowski, Adiabatic decomposition of the zetadeterminant and the Dirichlet to Neumann operator, J. Geom. Phys. 55 (2005), 241-266.

29. J. Park and K. P. Wojciechowski, Agranovich-Dynin formula for the zetadeterminants of the Neumann and Dirichlet problems, Spectral geometry of manifolds with boundary and decomposition of manifolds, 109-121, Contemp. Math., 366, Amer. Math. Soc., Providence, RI, 2005.

30. D. B. Ray and I. M. Singer, R-torsion and the Laplacian on Riemannian manifolds, Advances in Math. 7 (1971), 145-210.

31. S. G. Scott and K. P. Wojciechowski, The $\zeta$-determinant and Quillen determinant for a Dirac operator on a manifold with boundary, Geom. Funct. Anal. 10, no. 5 (1999), 1202-1236.

32. R. T. Seeley, Singular integrals and boundary value problems, Amer. J. Math. 88 (1966), 781-809.

33. R. T. Seeley, Topics in pseudo-differential operators, Pseudo-Diff. Operators (C.I.M.E., Stresa, 1968), 1969, pp. 167-305.

34. K. P. Wojciechowski, The additivity of the $\eta$-invariant. The case of a singular tangential operator, Comm. Math. Phys. 169 (1995), 315-327.

35. K. P. Wojciechowski, The $\zeta$-determinant and the additivity of the $\eta$-invariant on the smooth, self-adjoint Grassmannian, Comm. Math. Phys. 201, no. 2 (1999), 423-444. 